\title{
QCD Tests of the Puzzling Scalar Mesons
}

\author{
Stephan Narison ${ }^{\mathrm{a}}$

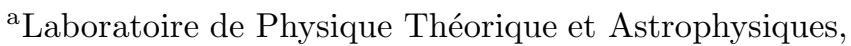 \\ Université de Montpellier II Place Eugène Bataillon, 34095 - Montpellier Cedex 05, France. \\ E-mail: snarison@yahoo.fr
}

\begin{abstract}
Motivated by several recent data, we test the QCD spectral sum rules (QSSR) predictions based on different proposals ( $\bar{q} q$, $\bar{q} \bar{q} q$, and gluonium) for the nature of scalar mesons. In the $I=1$ and $1 / 2$ channels, the unusual wrong splitting between the $a_{0}(980)$ and $\kappa(900)$ and the $a_{0}(980)$ width can be understood from QSSR within a $\bar{q} q$ assignement. However, none of the $\bar{q} q$ and $\bar{q} \bar{q} q q$ results can explain the large $\kappa$ width, which may suggest that it can result from a strong interference with non-resonant backgrounds. In the $I=0$ channel, QSSR and some low-energy theorems (LET) require the existence of a low mass gluonium $\sigma_{B}(1 \mathrm{GeV})$ coupled strongly to Goldstone boson pairs which plays in the $U(1)_{V}$ channel, a similar role than the $\eta^{\prime}$ for the value of the $U(1)_{A}$ topological charge. The observed $\sigma(600)$ and $f_{0}(980)$ mesons result from a maximal mixing between the gluonium $\sigma_{B}$ and $\bar{q} q(1 \mathrm{GeV})$ mesons, a mixing scheme which passes several experimental tests. OZI violating $J / \psi \rightarrow \phi \pi^{+} \pi^{-}, D_{s} \rightarrow 3 \pi$ decays and $J / \psi \rightarrow \gamma S$ glueball filter processes may indicate that the $f_{0}(1500), f_{0}(1710)$ and $f_{0}(1790)$ have significant gluonium component in their wave functions, while the $f_{0}(1370)$ is mostly $\bar{q} q$. Tests of these results can be provided by the measurements of the pure gluonium $\eta^{\prime} \eta$ and $4 \pi$ specific $U(1)_{A}$ decay channels.
\end{abstract}

\section{Introduction}

The nature of scalar mesons continues to be an intriguing problem in QCD. Experimentally, there are well established scalar mesons with isospin $I=1$, the $a_{0}(980)$, $a_{0}(1450)$ with isospin $I=1 / 2$, the $K_{0}^{*}(1410)$ meson, and with isospin $I=0$, the $f_{0}$-mesons at 980,1370 $[1,2]$ and $1500 \mathrm{MeV}$ from GAMS, CRYSTAL BARREL [1,2], WA102 [3] and BES [4]. Besides these resonances, there are different experimental indications [2], especially from BES [4], E791 [5], FOCUS [6], KLOE [7], SND [8], CMD2 [9], BELLE [10], WA102 [3] and $\pi \pi$ scattering data $[11,12]$ for some other scalar states, with $I=0$, the $\sigma(600), f_{0}(1710)$ and $f_{0}(1790)$, and with $I=1 / 2$, the $\kappa(840)$. The real quark and/or gluon contents of these states are not fully understood, which the interpretation using effective theories most of them based on a linear realization of chiral symmetry cannot clarify. In the following, we shall focus on the tests of the $\bar{q} q, \bar{q} \bar{q} q q$ and gluonium natures of these scalar mesons by confronting the recent experimental data with some QCD predictions based on QCD spectral sum rules (QSSR) complemented with some lowenergy theorems (LET) [13-16] and lattice calculations $[17,18]$.

\section{The $I=1,1 / 2$ scalar mesons}

\section{The $a_{0}(980)$ and $\kappa(840)$ masses}

These channels are expected to be simpler as we do not expect to have any mixing with a gluonium. If one assumes that these states are $\bar{q} q$ mesons, one can naturally associate them to the divergence of the vector currents:

$$
\begin{aligned}
a_{0}(980) & \rightarrow \partial_{\mu} V_{\bar{u} d}^{\mu} \equiv\left(m_{u}-m_{d}\right): \bar{u}(i) d:, \\
\kappa(840) & \rightarrow \quad \partial_{\mu} V_{\bar{u} s}^{\mu} \equiv\left(m_{u}-m_{s}\right): \bar{u}(i) s: .
\end{aligned}
$$

Within the QSSR approach, the properties and implications of these mesons can be studied from the two- point correlator:

$\psi_{\bar{u} q}\left(q^{2}\right)=i \int d^{4} x e^{i q x}\left\langle 0\left|\mathcal{T} \partial_{\mu} V_{\bar{u} q}^{\mu}(x) \partial_{\mu} V_{\bar{u} q}^{\mu}(0)^{\dagger}\right| 0\right\rangle$.

Since the pioneering work in [19], numerous authors have used and improved the analysis of the previous correlator for the extraction of the running $u$ - $d$ massdifference and strange quark masses $[13,20]$. The improvements come from the inclusion of higher order terms in the PT QCD series $[21,13]$; the inclusion of the $1 / q^{2}$ term $[22,23]$ which mimics the UV renormalon effects and which is also an alternative to the direct instanton effects where the later is not under a good quantitative control due to the incertainties of the instanton size and widths,...; the treatment of the spectral function using new $K \pi$ phase shift data [24]. The obtained value of the $u$ - $d$ mass-difference is consistent with some other determinations, while the extraction of the $a_{0}(980)$ mass from the sum rule $[13,20]$ is perfectly consistent with the data. Recent analysis [24] has lead to a value of the strange quark running mass which is consistent with some other QSSR determinations from different channels [25]. All these features support the $\bar{q} q$ meson assignements for the $a_{0}(980)$ and the $\kappa(840)$ mesons. However, the apparent wrong splitting of the $a_{0}(980)$ and the $\kappa(840)$ mesons is intriguing. Here, we investigate this analysis using the ratio of exponential Laplace/Borel sum rules:

$\mathcal{R}(\tau)=-\frac{d}{d \tau} \log \int_{0}^{\infty} d t e^{-t \tau} \operatorname{Im} \psi_{\bar{u} d}(t)$

Using the PT series to order $\alpha_{s}^{3}$, including the NP condensates of dimension 6 and the new $1 / q^{2}$ terms, we give the prediction for the $a_{0}(980)$ in Fig. 1a) and for the $\kappa(840)$ in Fig. 1b) using a NWA. We use the most recent value of $\bar{m}_{s}(2 \mathrm{GeV})=(96 \pm 5) \mathrm{MeV}$ compiled in [25]. One can see from these figures that the method reproduces the wrong splitting of the two mesons. The reason is that the $S U(3)$ breaking effects increase the 


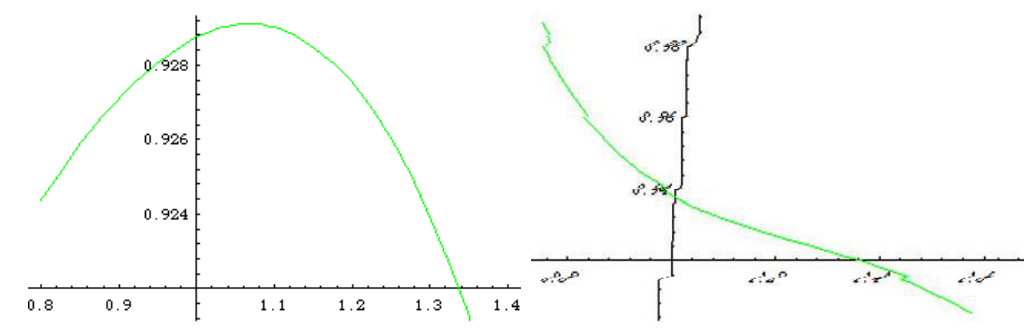

Figure 1. $\tau$ in $\mathrm{GeV}^{-2}$-dependence of the a) $M_{f\left(a_{0}\right)}$ in $\mathrm{GeV}$ and b) $M_{\kappa}$ in $\mathrm{GeV}$ for $\bar{m}_{s}(2 \mathrm{GeV})=98 \mathrm{MeV}$ at a given value of continuum threshold stability point: $t_{c} \approx 1 \mathrm{GeV}^{2}$.

value of the sum rule optimization scale compared to the one of the $a_{0}$ and then emphasize the contribution of the dimension six condensates, which is a vital correction in the analysis. Indeed, from the analytic expression of the sum rules, one can qualitatively extract the approximate mass formula ${ }^{1}$ :

$$
\begin{aligned}
M_{\kappa}^{2} & \simeq M_{a_{0}}^{2}+2 \bar{m}_{s}^{2}-8 \pi^{2} m_{s}\langle\bar{s} s\rangle \tau_{0} \\
& +\frac{3}{2} \frac{1408}{81} \pi^{3} \rho \alpha_{s}\left(\langle\bar{s} s\rangle^{2}-\langle\bar{u} u\rangle^{2}\right) \tau_{0}^{2} \\
& -\frac{1}{3} M_{\kappa}^{2} \Gamma_{\kappa}^{2} \tau_{0},
\end{aligned}
$$

where all different parameters including the $a_{0}$ mass are evaluated at the sum rule optimization scale $\tau_{0} \simeq$ $0.8 \mathrm{GeV}^{-2} ; \rho \simeq 2[29]$ indicates the deviation from the vacuum saturation of the four-quark condensate; $\langle\bar{s} s\rangle /\langle\bar{u} u\rangle \simeq 0.8$ measures the $S U(3)$ breaking of the quark condensate [13]. As shown in the above formula, the $S U(3)$ breaking corrections are relatively small, and the four-quark condensate tends to decrease the $\kappa$ mass ${ }^{2}$. Compared to the value of the $\kappa$ mass given in Fig. 1b) using NWA, the finite width correction reduces the mass by about $20 \mathrm{MeV}$, where we have used the width of $310 \mathrm{MeV}$ from BES [4]. One should mention that at the value of $\tau_{0}$, the OPE in powers of $\tau$ converges, while the radiative corrections to the parton graph, though large in individual sum rules, remain small in the ratio of moments $\mathcal{R}(\tau)$, as these corrections tend to compensate each others, justifying the uses of the result at this relativley low scale. From the previous analysis, we deduce:

$M_{a_{0}} \simeq 930 \mathrm{MeV} \quad$ and $\quad M_{\kappa} \simeq 920 \mathrm{MeV}$,

with about $10 \%$ error, in good agreement with recent data $[1,4,5]^{3}$.

\section{The decay constants}

The decay constant $f_{a_{0}}$ of the $a_{0}$ normalized as:

$\underline{\left\langle 0\left|\partial_{\mu} V_{\bar{u} d}^{\mu}\right| a_{0}\right\rangle \equiv \sqrt{2} f_{a_{0}} M_{a_{0}}^{2}}$,

${ }^{1}$ Notice that analogous formula in the vector channel explain with a relatively good accuracy the well-known $\phi-\rho$ and $K^{*}-\rho$ mass splittings [26].

${ }^{2}$ Using the instanton liquid model [27], the instanton contribution has been explicitly shown in [28] to be negligible.

${ }^{3}$ Previous sum rule analysis of the $\kappa$ parameters [13] used as in put a value of $m_{s}$ and $t_{c}$ much higher than here and gives higher value of the $\kappa$-mass. in the same way as $f_{\pi}=92.4 \mathrm{MeV}$ has been estimated several times in the literature $[13,30]$ :

$f_{a_{0}} \simeq(1.6 \pm 0.5) \mathrm{MeV}$,

where a better accuracy is claimed in [31]. Using $S U(3)$ symmetry and the almost degeneracy of the $a_{0}$ and $\kappa$ masses, we expect to have with a good accuracy:

$\frac{f_{\kappa}}{f_{a_{0}}} \simeq \frac{m_{s}-m_{u}}{m_{d}-m_{u}} \simeq 40$

\section{The hadronic couplings}

The $a_{0}$ and $\kappa$ hadronic couplings have been obtained using either a vertex sum rule [32,33] or/and $S U(3)$ symmetry rotation [34]. The leading order vertex sum rule results are:

$$
\begin{aligned}
& g_{a_{0} K^{+} K^{-}} \simeq \frac{8 \pi^{2}}{3 \sqrt{2}} \frac{m_{s}\langle\bar{s} s\rangle}{M_{K}^{2} f_{K}}\left(1-\frac{2}{r}\right) \simeq 3 \mathrm{GeV} \\
& \frac{g_{a_{0} K^{+} K^{-}}}{g_{\kappa K^{+} \pi^{-}}} \simeq e^{-\left(M_{K}^{2}-m_{\pi}^{2}\right) \tau_{0}}\left(1-\frac{2}{r}\right) \simeq 1.17,
\end{aligned}
$$

where we have used [13]: $m_{s}\langle\bar{s} s\rangle \simeq-0.8 M_{K}^{2} f_{K}^{2}, r \equiv$ $\langle\bar{s} s\rangle /\langle\bar{u} u\rangle \simeq 0.8$. and $\tau_{0} \simeq 1 \mathrm{GeV}^{-2}$. We expect an accuracy of about $20 \%$ (typical for the 3 -point fonction sum rules) for these estimates. Using the $S U(3)$ relation:

$g_{a_{0} \eta \pi} \simeq \sqrt{\frac{2}{3}} g_{a_{0} K^{+} K^{-}}$

one obtains:

$$
\begin{aligned}
\Gamma\left(a_{0} \rightarrow \eta \pi\right) & \simeq \frac{\left|g_{a_{0} \eta \pi}\right|^{2}}{16 \pi M_{a_{0}}}\left(1-\frac{M_{\eta}^{2}}{M_{a_{0}}^{2}}\right) \\
& \simeq 84 \mathrm{MeV},
\end{aligned}
$$

in agreement with the range of data from 50 to 100 $\mathrm{MeV}$ given by PDG [1]. Using the previous value of the $\kappa$ coupling, one can deduce:

$\Gamma(\kappa \rightarrow K \pi) \simeq \frac{3}{2} \Gamma\left(\kappa \rightarrow K^{+} \pi-\right) \simeq 104 \mathrm{MeV}$,

which is about a factor 4 smaller than the present data $[1,4]$, but is a typical value for the width of a $\bar{q} q$ state. The $\gamma \gamma$ widths

The $\gamma \gamma$ width of the $a_{0}$ has been evaluated using vertex 
sum rules within the $\bar{q} q$ and four-quark assignements of this meson, with the result $[30,34]$ :

$\Gamma a_{0}(\bar{q} q) \rightarrow \gamma \gamma \simeq(0.3 \sim 2.1) \mathrm{keV}$,

and [30]:

$\Gamma a_{0}(4 q) \rightarrow \gamma \gamma \simeq(2 \sim 5) \times 10^{-4} \mathrm{keV}$,

to be compared with the data of $(0.24 \pm 0.08) \mathrm{keV}$ compiled in [1]. Due to the inaccuracy of the QSSR predictions, we shall definitely use, in the following, the measured value of the $a_{0}$ width as an input for our theoretical predictions of the $\bar{q} q$ meson-width.

\section{Concluding remarks}

- The previous analysis shows that the mass and widths of the $a_{0}(980)$ are well described by a $\bar{q} q$ assignement of this meson.

- A QSSR analysis of the four-quark assignement [35] of these states gives predictions which reproduce the experimental mass of the $a_{0}(980)[36,30,13]$, like do the lattice calculations [37]. The result for the hadronic coupling $g_{a_{0} K^{+} K^{-}}$in the four-quark scenario depends crucially on the operators describing the $a_{0}$ and can range from $1.6 \mathrm{GeV}$ [38] to (5-8) GeV [30]. However, the prediction for the $\eta \pi$ can agree with the data [30] depending on the size of the operator mixing parameter. Therefore, an eventual selection of the two approaches will be a precise measurement of $g_{a_{0} K^{+} K^{-}}$ or/and a lattice measurement of the decay constant which should depend linearly on the light quark mass in the $\bar{q} q$ scheme, but is a constant in the four-quark one.

- Another problem arises when one computes the $\gamma \gamma$ width using vertex sum rules. The ratio of the widths in the two approaches is $[30]^{4}$ :

$\Gamma_{4 q \gamma \gamma} / \Gamma_{\bar{q} q \gamma \gamma} \approx(1 \sim 2) \times 10^{-3}$,

which is of the order of $\left(\alpha_{s} / p i\right)^{2}$, indicating that the four-quark assignement prediction is too small contrary to some claims in the literature.

- For the $\kappa$ meson, the $\bar{q} q$ assignement can reproduce quite well its wrong splitting with the $a_{0}$, but fails to reproduce its large experimental width [about a factor 4 smaller (see Eq. (12)], while the four-quark one gives a width of about a factor 2 smaller [38]. The failure of the two separate approaches ( $\bar{q} q$ and four-quark assignement) may suggest that the quark content of the $\kappa(841)$ is more complex than naïvely expected: it can be a mixing between a $\bar{q} q$ and a four-quark states, or it can come from a large interference of the $\bar{q} q$ ground state with non-resonant backgrounds. Further tests are needed for clarifying its nature.

\section{The $I=0$ bare scalar mesons}

The isoscalar scalar states are especially interesting in the framework of QCD since, in this anomalous $U(1)_{V}$ channel, their interpolating operator is the trace of the energy-momentum tensor:

$\theta_{\mu}^{\mu}=\frac{1}{4} \beta\left(\alpha_{s}\right) G^{2}+\sum_{i}\left[1+\gamma_{m}\left(\alpha_{s}\right)\right] m_{i} \bar{\psi}_{i} \psi_{i}$,

\footnotetext{
${ }^{4}$ We plan to come back to this point in a future work.
}

where $G_{\mu \nu}^{a}$ is the gluon field strengths, $\psi_{i}$ is the quark field; $\beta\left(\alpha_{s}\right) \equiv \beta_{1}\left(\alpha_{s} / \pi\right)+\ldots$ and $\gamma_{m}\left(\alpha_{s}\right) \equiv \gamma_{1}\left(\alpha_{s} / \pi\right)+$ ... are respectively the QCD $\beta$-function and quark mass-anomalous dimension $\left(\beta_{1}=-1 / 2(11-2 n / 3)\right.$ and $\gamma_{1}=2$ for $n$ flavours). In the chiral limit $m_{i}=0, \theta_{\mu}^{\mu}$ is dominated by its gluon component $\theta_{g}$, like is the case of the $\eta^{\prime}$ for the $U(1)_{A}$ axial-anomaly, explaining why the $\eta^{\prime}$-mass does not vanish like other Goldstone bosons for $m_{i}=0$. In this sense, it is natural to expect that these $I=0$ scalar states are glueballs/gluonia or have at least a strong glue component in their wave function. This gluonic part of $\theta_{\mu}^{\mu}$ should be identified with the $U(1)_{V}$ term [39] in the expression of the effective lagrangian based on a $U(3)_{L} \times U(3)_{R}$ linear realization of chiral symmetry (see e.g. $[40,41]$ ).

\section{Unmixed $\boldsymbol{I}=\mathbf{0}$ scalar $\bar{q} \boldsymbol{q}$ mesons}

We shall be concerned with the mesons $S_{2}$ and $S_{3}$ mesons associated respectively to the quark currents:

$J_{2}=m: \frac{1}{\sqrt{2}}(\bar{u} u+\bar{d} d): \quad$ and $\quad J_{3}=m_{s}: \bar{s} s:$.

From the good realization of the $S U(2)$ flavour symmetry $\left(m_{u}=m_{d}\right.$ and $\left.\langle\bar{u} u\rangle=\langle\bar{d} d\rangle\right)$, one expects a degeneracy between the $a_{0}$ and $S_{2}$ states:

$M_{S_{2}} \simeq M_{a_{0}} \simeq 930 \mathrm{MeV}$

while its hadronic coupling is $[34,15]$ :

$g_{S_{2} \pi^{+} \pi^{-}} \simeq \frac{16 \pi^{3}}{3 \sqrt{3}}\langle\bar{u} u\rangle \tau_{0} e^{M_{2}^{2} \tau_{0} / 2} \simeq 2.46 \mathrm{GeV}$

corresponding to ${ }^{5}$ :

$$
\Gamma\left(S_{2} \rightarrow \pi^{+} \pi^{-}\right) \simeq 120 \mathrm{MeV},
$$

Using $S U(3)$ symmetry, one can also deduce:

$g_{S_{2} K^{+} K^{-}} \simeq \frac{1}{2} g_{S_{2} \pi^{+} \pi^{-}} \simeq 1.23 \mathrm{GeV}$.

The $S_{2} \gamma \gamma$ width can be deduced from the one of the $a_{0}(\bar{q} q)$ obtained previously, through the non-relativistic relation (ratio of the square of quark charges):

$\Gamma_{S_{2} \rightarrow \gamma \gamma} \simeq \frac{25}{9} \Gamma_{a_{0} \rightarrow \gamma \gamma} \simeq(0.7 \pm 0.2) \mathrm{keV}$.

The mass of the mesons containing a strange quark is predicted to be [15]:

$M_{S_{3}} / M_{\kappa} \simeq 1.03 \pm 0.02 \Longrightarrow M_{S_{3}} \simeq 948 \mathrm{MeV}$,

if one uses $M_{\kappa}=920 \mathrm{MeV}^{6}$, while its coupling to $K^{+} K^{-}$is [15]:

$$
g_{S_{3} K^{+} K^{-}} \simeq(2.7 \pm 0.5) \mathrm{GeV} .
$$

These results suggest that the naïve $\bar{q} q$ assignement of the $\sigma(600) \equiv S_{2}$ does not fit the data.

${ }^{5}$ We use the normalization:

$$
\Gamma\left(\sigma_{B} \rightarrow \pi \pi\right)=\frac{3}{2} \frac{\left|g_{\sigma_{B} \pi+\pi-}\right|^{2}}{16 \pi M_{\sigma_{B}}}\left(1-\frac{4 m_{\pi}^{2}}{M_{\sigma_{B}}^{2}}\right)^{1 / 2} .
$$

${ }^{6}$ In [15] a higher value has been obtained because one has used as input the experimental mass $K_{0}^{*}=1430 \mathrm{MeV}$. 


\section{Gluonia masses and decay constants}

- These states have been explicitly analyzed in $[16,42$, 15] using QSSR of the two-point correlator:

$\psi_{s}\left(q^{2}\right)=16 i \int d^{4} x e^{i q x}\left\langle 0\left|\mathcal{T} \theta_{\mu}^{\mu}(x) \theta_{\mu}^{\mu}(0)^{\dagger}\right| 0\right\rangle$,

in the chiral limit $m_{q}=0$, for the observables:

$\mathcal{L}_{n}(\tau)=-\int_{0}^{\infty} d t t^{n} e^{-t \tau} \operatorname{Im} \psi_{s}(t)$,

and:

$\mathcal{R}_{n, n+1}(\tau)=-\frac{d}{d \tau} \log \int_{0}^{\infty} d t t^{n} e^{-t \tau} \operatorname{Im} \psi_{s}(t)$,

where $n=-1,0,1,2$. For $n=-1$, the sum rule is sensitive to the subtraction constant:

$\psi_{s}(0)=-16 \frac{\beta_{1}}{\pi}\left\langle\alpha_{s} G^{2}\right\rangle$,

fixed from LET [43], where $\left\langle\alpha_{s} G^{2}\right\rangle=(0.07 \pm 0.01)$ $\mathrm{GeV}^{4}[44,13]$. One has found in $[42]$ that, due to $\psi_{s}(0)$, the subtracted sum rule (SSR) $\mathcal{L}_{-1}$ is more weighted by the high-energy behaviour of the spectral integral than the unsubtracted sum rule (USR) $\mathcal{L}_{0,1,2}$, which motivated the introduction of 2 resonances (below and above $1 \mathrm{GeV}$ ) for solving the controversial results obtained in the past. The results of the analysis using the standard OPE by retaining higher order PT series and the lowest dimension condensates are $[16,42,15]$ :

$$
\begin{aligned}
f_{\sigma_{B}} & \simeq(884 \pm 116) \mathrm{MeV}, \\
f_{G} & \simeq(390 \pm 145) \mathrm{MeV},
\end{aligned}
$$

corresponding to:

$M_{\sigma_{B}} \simeq 1 \mathrm{GeV} \quad$ and $\quad M_{G} \simeq(1.5 \pm 0.2) \mathrm{GeV}$.

$M_{\sigma_{B}}$ has been obtained in [16] using a least square fit of $\mathcal{R}_{0,1}$. Its decay constant has been obtained using a least square fit of $\mathcal{R}_{0,1}$ or/and a stability criterion of $\mathcal{L}_{0}$ [42] ${ }^{7}$. The mass of the 2 nd resonance has been fixed from $\mathcal{R}_{0,1}[15]$ and its decay constant comes from $\mathcal{L}_{-1}$ [42]. We shall see that $M_{G}$ is the one which can be compared with the present lattice value of about $1.6 \mathrm{GeV}$ in the quenched approximation [17], while the $\sigma_{B}$ mass will be a $\eta^{\prime}$-like meson expected to couple strongly with Goldstone boson pairs (huge OZI violation) $[42,15]$ and playing a role in the saturation of the $U(1)_{V}$ two-point correlator subtraction constant $\psi_{s}(0)$. It can only be tested using lattice with dynamical fermions (see e.g. [18] for the inclusion of the disconnected part of the scalar propagator).

- One can also notice that a possible effect of the radial excitation of the $\sigma$ can be obtained by matching the radial excitation contribution with the QCD continuum. Assuming its mass to be around $1.4 \mathrm{GeV}$, one can deduce [42]:

$f_{\sigma_{B}^{\prime}} \leq(139 \sim 224) \mathrm{MeV}$,

${ }^{7}$ A similar analysis in the $U(1)_{A}$ channel has given an estimate of the $\eta^{\prime}$ parameters and of the $U(1)_{A}$ topological charge $[45,16]$. while a weaker bound of about $500 \mathrm{MeV}$ has been allowed in [15].

- The effect of the $1 / q^{2}$ term, to the previous results, which is an alternative of the direct instanton contribution has been shown to be small [22], though this term is necessary for solving the sum rule scale hierarchy of the gluonia channels compared to the usual $\bar{q} q$ mesons.

- A recent QSSR analysis of the same gluonium correlator using Gaussian sum rules and including instantons [46] confirms the previous mass values obtained in Eq. (30), but not the results in ref. [47,48], where it is argued that the presence of the direct instantons solve the controversial results noticed in $[42,15]$ between the subtracted $n=-1$ sum rule with the other $n \geq 0$ unsubtracted ones, without the need of two resonances. In our normalization, the results in [47] are:

$M_{S}=(1.25 \pm 0.2) \mathrm{GeV}, \quad f_{S}=(3 \pm 0.3) \mathrm{GeV}$.

The mass value ${ }^{8}$ does not contradict the ones in Eq. (30) as it is about the mean value of the two resonances ones, while the decay constant leads to $[43,42,15]$ :

$B(J / \psi \rightarrow S \gamma) \simeq 1.5 \times 10^{-2}$,

which is about 10 times higher than the one of the $J / \psi \rightarrow f_{2}(1.24) \gamma$ and which is already excluded by the BES [4] and some other data. This fact may signal some eventual internal inconsistencies in the treatment of the instanton contributions.

- An upper bound on the gluonium mass has been also obtained in [15]:

$M_{G} \leq(2.16 \pm 0.22) \mathrm{GeV}$,

using the positivity of the moment $\mathcal{R}_{1,2}$. This bound has been strengthened in [47] to $1.7 \mathrm{GeV}$, which cannot be an absolute bound because of the inclusion of the QCD continuum model and of its related uncertainties for its derivation.

Gluonia widths to $\pi \pi$

- For this purpose, we consider the gluonium- $\pi \pi$ vertex:

$V\left(q^{2}\right)=\left\langle\pi_{1}\left|\theta_{\mu}^{\mu}\right| \pi_{2}\right\rangle, \quad q=p_{1}-p_{2}$,

where: $V(0)=2 m_{\pi}^{2}$. In the chiral limit $\left(m_{\pi}^{2} \simeq 0\right)$, the vertex obeys the dispersion relation:

$V\left(q^{2}\right)=\int_{0}^{\infty} \frac{d t}{t-q^{2}-i \epsilon} \frac{1}{\pi} \operatorname{Im} V(t)$,

which gives the 1st NV sum rule [42]:

$\frac{1}{4} \sum_{S \equiv \sigma_{B}, \sigma_{B}^{\prime}, G} g_{S \pi \pi} \sqrt{2} f_{S} \simeq 0$.

Using the fact that $V^{\prime}(0)=1$ [49], one obtains the 2nd NV sum rule [42]:

$\frac{1}{4} \sum_{S \equiv \sigma_{B}, \sigma_{B}^{\prime}, G} g_{S \pi \pi} \sqrt{2} f_{S} / M_{S}^{2}=1$.

To a first approximation, we assume a $\sigma_{B}$-dominance in the 2nd NV sum rule, while in the 1st sum rule, there is

${ }^{8} \mathrm{It}$ is an upper bound in [48]. 
a matching between the $\sigma_{B}$ and all higher mass glueball contributions, which we replace by an effective $\sigma^{\prime}$ mass of about $1.4 \mathrm{GeV}$. Then, one obtains:

$g_{\sigma_{B} \pi^{+} \pi^{-}} \simeq g_{\sigma_{B} K^{+} K^{-}} \simeq(3.2 \sim 6.8) \mathrm{GeV}$.

A complete matching in the 1st NV sum rule would lead to an effective coupling:

$g_{\sigma_{B}^{\prime} \pi^{+} \pi^{-}} \approx 12 \mathrm{GeV}$,

when using $f_{\sigma}^{\prime} \simeq 500 \mathrm{MeV}$. An interpretation of this value of $g_{\sigma_{B}^{\prime} \pi \pi}$ is unclear but it is expected to parametrize all higher states contributions to the 1st sum rule. If one uses a resonance mass of about 1.4 $\mathrm{GeV}$, one would obtain a very broad $\sigma_{B}^{\prime}$ which can mimic the red dragon proposed earlier [50], but cannot manifest as a peak, making its identification with a true resonance difficult. Different strategies for extracting the $f_{0}(1370)$ parameters from the continuum background has been discussed in [12] leading to a width of the $f_{0}$ compiled in PDG [1]. For definiteness, we shall use in the following, the value of $\Gamma\left(\sigma^{\prime} \rightarrow \pi \pi\right.$ about $250 \mathrm{MeV}$ obtained in [12] within a factor two accuracy. This leads to:

$g_{\sigma_{B}^{\prime} \pi^{+} \pi^{-}} \simeq g_{\sigma_{B}^{\prime} K^{+} K^{-}} \simeq(4.2 \pm 0.7) \mathrm{GeV}$.

$\sigma_{B}$ as an $\eta^{\prime}$-like meson

- One can check that the LET for $\psi_{s}(0)$ given in Eq. (28) is almost saturated by the contribution of the lowest mass $\sigma_{B}(1$.) meson:

$\psi_{s}(0) \simeq 2 M_{\sigma_{B}}^{2} f_{\sigma_{B}}^{2} \simeq 1.5 \mathrm{GeV}^{4}$,

compared to the LET value of $1.6 \mathrm{GeV}^{4}$. This property is very similar to the contribution of the $\eta^{\prime}$ in the topological charge (subtraction constant of the anomalous $U(1)_{A}$ gluonium two-point correlator [51]), which explains why it is not degenerated with the pion at finite $N_{c}$.

- The $\sigma_{B}$ large coupling to pseudoscalar pairs, through OZI violating process, can also be compared with the affinity of the $\eta^{\prime}$ to couple to ordinary mesons, making it as an ambidextre gluonium-meson state

- Like in the $U(1)_{A}$ sector, the quenched lattice simulations obtain a higher glueball mass which is not the $\eta^{\prime}$ mass. The $\eta^{\prime}$-mass and decay constant are only measured from a lattice calculation of the $U(1)_{A}$ topological charge [52]. We expect that the same situation occurs in the $U(1)_{V}$ channel, where a quenched lattice gives a scalar gluonium mass of about $1.5 \mathrm{GeV}$, while the $\sigma_{B}$ parameters can be obtained from the measurement of the scalar correlator subtraction constant $\psi_{s}(0)$ including dynamical fermions.

$G(1.5)$ widths into $\eta^{\prime} \eta^{\prime}, \eta \eta^{\prime}$ and $\eta \eta$

- Analogous low-energy theorem [42] gives:

$\left\langle\eta_{1}\left|\theta_{\mu}^{\mu}\right| \eta_{1}\right\rangle=2 M_{\eta_{1}}^{2}$,

where $\eta_{1}$ is the unmixed $U(1)$ singlet state of mass $M_{\eta_{1}} \simeq 0.76 \mathrm{GeV}$ [51]. Writing the dispersion relation for the vertex, one obtains the NV sum rule:

$\frac{1}{4} \sum_{S \equiv \sigma_{B}, \sigma_{B}^{\prime}, G} g_{S \eta_{1} \eta_{1}} \sqrt{2} f_{S}=2 M_{\eta_{1}}^{2}$, which, by assuming a $G$-dominance of the vertex sum rule, leads to:

$g_{G \eta_{1} \eta_{1}} \approx(1.2 \sim 1.7) \mathrm{GeV}$.

Introducing the "physical" $\eta^{\prime}$ and $\eta$ through:

$\eta^{\prime} \sim \cos \theta_{P} \eta_{1}-\sin \theta_{P} \eta_{8}$

$\eta \sim \sin \theta_{P} \eta_{1}+\cos \theta_{P} \eta_{8}$,

where $[1,53] \theta_{P} \simeq-(18 \pm 2)^{\circ}$ is the pseudoscalar mixing angle, one can deduce:

$\Gamma\left(G \rightarrow \eta^{\prime} \eta\right) \simeq(5 \sim 10) \mathrm{MeV}$.

The previous scheme is also known to predict (see NV and [59]):

$r \equiv \frac{\Gamma_{G \eta \eta}}{\Gamma_{G \eta \eta^{\prime}}} \simeq 0.22, \quad g_{G \eta \eta} \simeq \sin \theta_{P} g_{G \eta \eta^{\prime}}$,

compared with the GAMS data [1] $r \simeq 0.34 \pm 0.13$. This result can then suggest that the $G(1.6)$ seen by the GAMS group is a pure gluonium, which is not the case of the particle seen by Crystal Barrel [1] which corresponds to $r \approx 1$.

\section{Gluonia widths into $4 \pi$}

Within our scheme, we expect that the $4 \pi$ are mainly $S$-waves initiated from the decay of pairs of $\sigma_{B}$. Using:

$\left\langle\sigma_{B}\left|\theta_{\mu}^{\mu}\right| \sigma_{B}\right\rangle=2 M_{\sigma_{B}}^{2}$,

and writing the dispersion relation for the vertex, one obtains the sum rule:

$\frac{1}{4} \sum_{i=\sigma_{B}, \sigma_{B}^{\prime}, G} g_{S \sigma_{B} \sigma_{B}} \sqrt{2} f_{S}=2 M_{\sigma_{B}}^{2}$.

We use $M_{\sigma_{B}} \simeq 0.6 \sim 1 \mathrm{GeV}, M_{\sigma_{B}^{\prime}} \simeq 1.4 \mathrm{GeV}$ and the observed $f_{0}(1.37)$ width into $4 \pi$ of about $(106 \sim 250)$ $\mathrm{MeV}$ [1] ( $S$-wave part). Neglecting, to a first approximation, the $\sigma_{B}$ contribution to the sum rule, we can deduce:

$\left|g_{G \sigma_{B} \sigma_{B}}\right| \approx 1.3 \sim 3.7 \mathrm{GeV}$,

where the first (resp. second) value corresponds to $M_{\sigma_{B}} \simeq 0.6 \mathrm{GeV}$ (resp. $1 \mathrm{GeV}$ ). This leads to the width into $\sigma(600) \sigma(600)$ of about $(7-55) \mathrm{MeV}$, much larger than the one into $\eta \eta$ and $\eta \eta^{\prime}$. This feature is satisfied by the $G(1.5)$ state seen by GAMS, Crystal Barrel and WA102 [1]. However, the previous approaches show the consistency in interpreting the $G(1.5)$ seen at GAMS as an "almost" pure gluonium state (ratio of the $\eta \eta^{\prime}$ versus the $\eta \eta$ widths), while the state seen by the Crystal Barrel and WA102, though having a gluon component in its wave function, cannot be a pure gluonium because of its prominent decays into $\eta \eta$ and $\pi^{+} \pi^{-}$.

Gluonia widths into $\gamma \gamma$

These widths have been derived in [42] by identifying the $\gamma \gamma$-glue-glue box diagram with the scalar $\gamma \gamma \mathrm{La}$ grangian where the quarks in the internal have been taken to be non-relativistic. In this way, one has obtained:

$g_{\sigma \gamma \gamma} \simeq \frac{\alpha}{60} \sqrt{2} f_{\sigma} M_{\sigma}^{2}\left(\frac{\pi}{-\beta_{1}}\right) \sum_{u, d, s} Q_{i}^{2} / m_{i}^{4}$, 
where $Q_{i}$ and $m_{i}$ are the quark charge and constituent masses. This leads to:

$$
\begin{aligned}
\Gamma\left(\sigma_{B} \rightarrow \gamma \gamma\right) & \simeq(0.03 \sim 0.08) \mathrm{keV}, \\
\Gamma\left(\sigma_{B}^{\prime} \rightarrow \gamma \gamma\right) & \simeq(0.01 \sim 0.03) \mathrm{keV}, \\
\Gamma(G \rightarrow \gamma \gamma) & \simeq(0.3 \sim 0.6) \mathrm{keV} .
\end{aligned}
$$

Alternatively, one can use the trace anomaly to order $k^{4}$ in order to deduce:

$\left\langle 0\left|\frac{1}{4} \beta\left(\alpha_{s}\right) G^{2}\right| \gamma_{1} \gamma_{2}\right\rangle \simeq-\left\langle 0\left|\left(\frac{\alpha}{3 \pi}\right) R F_{1}^{\mu \nu} F_{2}^{\mu \nu}\right| \gamma_{1} \gamma_{2}\right\rangle$,

where $R \equiv 3 \sum_{i} Q_{i}^{2}, \alpha$ is the QED coupling. This relation gives:

$\frac{\sqrt{2}}{4} \sum_{\sigma, \ldots} f_{i} g_{i \gamma \gamma} \simeq\left(\frac{\alpha}{3 \pi}\right) R$.

From this relation and using the previous values of $f_{\sigma}$ and $f_{\sigma}^{\prime}$, one can deduce:

$\Gamma_{G \rightarrow \gamma \gamma} \simeq(1 \sim 6) \mathrm{keV}$,

which is quite inaccurate but still consistent with the previous determination.

\section{Comments}

Comparing the above results, especially the predicted widths, with the experimentally observed candidates, it is likely that the $\sigma$ and some of its radial excitations have a lot of glue in their wave functions. As a consequence, a quarkonium-gluonium decay mixing scheme ${ }^{9}$ has been proposed in the $I=0$ scalar sector. [34,15], for explaining the observed spectrum and widths of the possibly wide $\sigma(<1 \mathrm{GeV})$ and the narrow $f_{0}(980)^{10}$.

\section{Meson-gluonium mixing below $1 \mathrm{GeV}$}

- BES data suggest that the $\sigma(600)$ is produced in the OZI forbidden $J / \psi \rightarrow \phi \pi^{+} \pi^{-}$process [4], which can indicate the large amount of glue in its wave function. Its production from the OZI allowed $J / \psi \rightarrow$ $\omega \pi^{+} \pi^{-}, K^{+} K^{-}$processes, is expected to be due to its quark component, while the relative small branching ratio in the OZI allowed $J / \psi \rightarrow \phi K^{+} K^{-}$process relative to $J / \psi \rightarrow \phi \pi^{+} \pi^{-}$can be due to an interference between the $K^{+} K^{-}$amplitude from the gluon and quark components of the $\sigma$.

- In the same way, the $f_{0}(980)$ is produced in the OZI violating $J / \psi \rightarrow \phi \pi^{+} \pi^{-}$, [4] and $D_{s} \rightarrow \pi^{-} \pi^{+} \pi^{+}$[5] processes which may also indicate its gluonium component, while its production from $J / \psi \rightarrow \phi K^{+} K^{-}$, can signal a strong $\bar{s} s$ component in its wave function. We shall keep in mind these results for building the mixing scheme.

\section{The meson-gluonium mixing scheme}

- We assume that the observed states come from the

\footnotetext{
${ }^{9}$ This has to be contrasted with the small mass-mixing coming from the off-diagonal two-point function [54].

${ }^{10}$ We shall not consider in our analysis the recent result of [55] from $\gamma \gamma \rightarrow \pi^{0} \pi^{0}$ where the resulting $\sigma \rightarrow \gamma \gamma$ width of about 4 $\mathrm{keV}$ is much bigger than generally expected and which needs to be confirmed by some other data. We plan to come back to this point in the future.
}

mixing between the gluonium $\sigma_{B}$ and quark $S_{2} \equiv$ $1 / \sqrt{2}(\bar{u} u+\bar{d} d)$ and $S_{3} \equiv \bar{s} s$ bare states ${ }^{11}$ :

$\left(\begin{array}{c}\sigma \\ f_{0}\end{array}\right)=\left(\begin{array}{cc}\cos \theta_{S} & \sin \theta_{S} \\ -\sin \theta_{S} & \cos \theta_{S}\end{array}\right)\left(\begin{array}{c}\sigma_{B} \\ S_{2}+\phi_{S} S_{3}\end{array}\right)$

where $\phi_{S}=1 / \sqrt{2}$ for a $S U(3)$ singlet and $-\sqrt{2}$ for an $\mathrm{SU}(3)$ octet.

- In [34], the mixing angle $\theta_{S}$ has been fixed from the analysis of the predicted decays of the hypothetical bare states $S_{2}, \sigma_{B}$ and of the observed meson $f_{0}(980) \rightarrow \gamma \gamma$. Using the predictions: $\Gamma\left(S_{2} \rightarrow \gamma \gamma\right)$ in Eq. (22), $\Gamma\left(\sigma_{B} \rightarrow \gamma \gamma\right) \simeq 0.03 \mathrm{keV}$ [42], and the recent data $\Gamma\left(f_{0}(980) \rightarrow \gamma \gamma\right) \simeq(0.4 \pm 0.1) \mathrm{keV}[1]$, one can deduce:

$\theta_{S} \simeq(45 \pm 15)^{0}$,

where the $S_{3} \rightarrow \gamma \gamma$ width is suppressed as $(2 / 25)$ compared to the one of the $S_{2}$.

- Using, for definiteness, as inputs the theoretical predictions given in Eqs (19) and (39), we predict the couplings:

$\begin{aligned} g_{\sigma \pi^{+} \pi^{-}} & \simeq(5.3 \pm 1.8) \mathrm{GeV} \\ g_{f_{0} \pi^{+} \pi^{-}} & \simeq(1.8 \pm 1.3) \mathrm{GeV}\end{aligned}$

in reasonnable agreement with the ones from the data:

$g_{\sigma \pi^{+} \pi^{-}}^{\exp } \simeq(3.0 \pm 1.5) \mathrm{GeV}$,

$g_{f_{0} \pi^{+} \pi^{-}}^{\exp } \simeq(1.5 \pm 0.3) \mathrm{GeV}$,

corresponding to:

$$
\begin{aligned}
\Gamma^{e x p}(\sigma(600) \rightarrow \pi \pi) & \approx 481 \mathrm{MeV}, \\
\Gamma^{\exp }\left(f_{0}(980) \rightarrow \pi \pi\right) & \approx(70 \pm 30) \mathrm{MeV},
\end{aligned}
$$

- In order to predict the mixing parameter $\phi_{S}$, we fit the experimental $f_{0} K^{+} K^{-}$width from BES [4]:

$g_{f_{0} K^{+} K^{-}} / g_{f_{0} \pi^{+} \pi^{-}} \simeq 2.05 \pm 0.15$,

and we use the theoretical predictions given in Eqs (19), (24) and (39). Then, we obtain:

$\phi_{S} \simeq 3.0, \quad$ and $\quad g_{\sigma K^{+} K^{-}} / g_{\sigma \pi^{+} \pi^{-}} \simeq 2$.

Further data are needed for improving and testing this result.

- One should note that using only the constraint from the $f_{0} \rightarrow \pi^{+} \pi^{-}$and $f_{0} \rightarrow K^{+} K^{-}$hadronic widths, one would obtain:

$\theta_{S}=16^{0}, \quad$ and $\quad \phi_{S} \simeq-1.4$

indicating that the $\sigma$ is an almost pure gluonium and the $f_{0}$ a $\bar{q} q S U(3)$ octet, which is similar to the scheme in [50]. However, the result in Eq. (63) would give a too high value of $f_{0} \rightarrow \gamma \gamma$ and does not explain the OZI violating production of the $f_{0}$ in $J / \psi$ and $\phi$ radiative

\footnotetext{
${ }^{11}$ In our approach, we first calculate the real part of the masses of these hypothetical states and deduce their widths using vertex sum rules. These states would correspond to the bare states in the $K$-matrix formalism (see e.g. [12]). Due to the large error in our mass predictions, we neglect, to a first approximation, some possible shifts on the masses which can be induced by the decay processes mentioned in [12].
} 
decays.

Comments on alternative approaches

- One should note that a four-quark QSSR analysis gives [38]:

$\begin{aligned} g_{f_{0} K^{+} K^{-}} & \simeq g_{a_{0} K^{+} K^{-}} \simeq(1.6 \pm 0.1) \mathrm{GeV} \\ g_{f_{0} \pi^{+} \pi^{-}} & \simeq(0.47 \pm 0.05) \mathrm{GeV}\end{aligned}$

where the absolute values differ from the ones given in [56] and then question the realibility of the results obtained there. Therefore, lattice calculations of these couplings become mandatory. Eq. (64) leads to:

$\Gamma\left(f_{0}(980) \rightarrow \pi \pi\right) \approx 7 \mathrm{MeV}$,

which is too small compared with the range $(40 \sim 100)$ $\mathrm{MeV}$ given by the data [1]

- Alternative approaches based on $\bar{K} K$ loop, $\bar{K} K$ molecules and four-quark states can predict value of $\Gamma\left(f_{0}(980) \rightarrow \gamma \gamma\right)$ in agreement with the data [57],[58], but, most of them, do not give a satisfactory prediction for the $f_{0} \rightarrow \pi^{+} \pi^{-}$width and their production from OZI violating decays. On the other, it would be interesting to see the connection of these effective approaches with the quark-gluon picture used here.

Tests from $J / \psi$ and $\phi$ radiative decays.

- These decays are known to be a gluonium filter. The production of a gluonium $S$ from $J / \psi$ radiative decays can be approximated by [49]:

$$
\begin{gathered}
\Gamma(J / \psi \rightarrow \gamma S) \simeq \frac{\alpha^{3} \pi}{\beta_{1}^{2} 656100}\left(\frac{M_{J / \psi}}{M_{c}}\right)^{4}\left(\frac{M_{S}}{M_{c}}\right)^{4} \\
\frac{\left(1-M_{S}^{2} / M_{J / \psi}^{2}\right)^{3}}{\Gamma\left(J / \psi \rightarrow e^{+} e^{-}\right)} f_{S}^{2}
\end{gathered}
$$

where $M_{c} \simeq 1.5 \mathrm{GeV}$ is the charm constituent quark mass. In our scheme, the $\sigma$ is mostly a gluonium. Therefore, one expects the branching ratio:

$B(J / \psi \rightarrow \gamma \sigma) \approx 19 \times 10^{-5}$.

Extrapolating the previous exppression to the $\phi$-meson and using $M_{s} \simeq 500 \mathrm{MeV}$, one obtains:

$B(\phi \rightarrow \gamma \sigma) \approx 12 \times 10^{-5}$,

which, despite the crude approximation used, compares quite well with the KLOE [7] data:

$B\left(\phi \rightarrow \gamma \pi^{0} \pi^{0}\right) \approx(10.9 \pm 0.3 \pm 0.5) \times 10^{-5}$.

Tests from $D_{(s)}$ semileptonic decays

This section has been discussed in details in [28] and will be only sketched in the following.

- $S_{2}(\bar{u} u+\bar{d} d)$ meson productions

If the scalar mesons were simple $\bar{q} q$ states, the semileptonic decay width could be calculated quite reliably using QSSR, where the relevant diagram is a quark loop triangle. Several groups [60] predict all form factor to be: $f_{+}(0) \approx 0.5$, yielding, for $M_{S_{2}} \simeq 600 \mathrm{MeV}$, a decay rate:

$\Gamma\left(D \rightarrow S_{2} l \nu\right)=(8 \pm 3) 10^{-16} \mathrm{GeV}$,

which is, unfortunately, even in high stastistics experiments, at the edge of observation since the decays into an isoscalar are CKM-suppressed due to the $c$ - $u$ transition at the weak vertex.

- Scalar gluonium or/and $\bar{s} s$ productions

Semi-qualitative but model independent results for the production of gluonium have been given in [28] (see also [61]):

- The only way to obtain a non-CKM suppressed isoscalar is to look at the semileptonic decay of the $D_{s}$-meson, where the light quark is a strange one and an isoscalar $s \bar{s}$ or/and gluonium state can be formed.

- If the $\bar{s} s$ state is relatively light $(<1 \mathrm{GeV})$, which might be the natural partner of the $(\bar{u} u+\bar{d} d)$ often interpreted to be a $\sigma(600)$ in the literature, then, one should produce a $K \bar{K}$ pair through the isoscalar $\bar{s} s$ state. The non-observation of this process will disfavour the $\bar{q} q$ interpretation of the $\sigma$ and $f_{0}$ mesons.

- If a gluonium state is formed it will decay with even strength into $\pi \pi$ and a $K \bar{K}$ pairs. Therefore a gluonium formation in semileptonic $D_{s}$ decays should result in the decay patterns:

$D_{s} \rightarrow \sigma_{B} \ell \nu \rightarrow \pi \pi \ell \nu \quad D_{s} \rightarrow \sigma_{B} \ell \nu \rightarrow K \bar{K} \ell \nu$,

with about the same rate up to phase space factors. The observation of the semileptonic $\pi \pi$ decay of the $D_{s}$ by E791 [5] is a sign for glueball formation ${ }^{12}$.

- Using, e.g., the result in [60], the one for light $S(\bar{q} q)$ quarkonium production behaves as:

$\Gamma\left[D_{s} \rightarrow S_{q} l \nu\right] \sim\left|V_{c q}\right|^{2} G_{F}^{2} M_{c}^{5}\left|f_{+}(0)\right|^{2}$.

- The $\sigma_{B}(g g)$ production, can be obtained from the $1 / M_{c}$ behaviour of the $W W g g$ box diagram. Using dispersion techniques similar to the one used for $J / \psi \rightarrow$ $\sigma_{B} \gamma$ processses $[43,42,13]$, one obtains, assuming a $D_{s}$ and $\sigma_{B}$-dominances [28]:

$\Gamma\left[D_{s} \rightarrow \sigma_{B} l \nu\right] \sim\left|V_{c s}\right|^{2} G_{F}^{2} \frac{\left|\left\langle 0\left|\phi_{S} G^{2}\right| \sigma_{B}\right\rangle\right|^{2}}{M_{c} M_{\sigma}^{4}}$,

where $\left\langle 0\left|\phi_{S} G^{2}\right| \sigma_{B}\right\rangle$ is by definition proportional to $f_{\sigma} M_{\sigma}^{2}$. Then, one deduces:

$\frac{\Gamma\left[D_{s} \rightarrow \sigma_{B}(g g) l \nu\right]}{\Gamma\left[D_{s} \rightarrow S_{q}(\bar{q} q) l \nu\right]} \sim \frac{1}{\left|f_{+}(0)\right|^{2}}\left(\frac{f_{\sigma}}{M_{c}}\right)^{2}$,

which is $\mathcal{O}(1)$ for $f_{\sigma} \simeq 0.8 \mathrm{GeV}$.

\section{- Comments}

These semi-quantitative results indicate that the gluonium production rate can be of the same order as the $\bar{q} q$ one contrary to the naïve perturbative expectation $\left(\alpha_{s}^{2}\right.$ suppression), which is a consequence of the OZI-rule violation of the $\sigma_{B}$ decay. ${ }^{13}$ However, it also shows that, due to the (almost) universal coupling of the $\sigma_{B}$ to Goldstone boson pairs, one also expects a production of the $K \bar{K}$ pairs, which can compete with the one from $\bar{s} s$ quarkonium state, and again renders more difficult the identification of the such $\bar{s} s$ state if allowed by phase space.

$\overline{12}$ An alternative explanation assuming $f_{0}(\bar{s} s)$ and using $\bar{K} K$ loop has been given in [58]. However, the same assumption for $f_{0}$ but using QSSR leads to a negative conclusion [62].

${ }^{13}$ Productions of the scalar mesons in $B$-decays have been discussed in [63]. 


\section{Properties of the mesons above $1 \mathrm{GeV}$}

QSSR does not have a precise systematic framework for extracting the properties of the radial excitations, except the approximate value of the mass indicated by the value of the QCD continuum threshold at which the mass of the ground state has been optimized. In order to check this result, one often uses (if available) empirical observations of the splittings between the radial excitations and the ground state, or/and arguments based on the (linear) Regge trajectories. For this reason, the discussions which we shall give below will be very qualitative.

\section{The $I=1$ and $1 / 2$ mesons}

The $a_{0}(1450)$ and the $K_{0}^{*}(1430)$ are almost degenerated indicating the restoration of the $S U(3)$ flavour symmetry where the $S U(3)$ breakings behaves like $m_{s}^{2} / M_{R}^{2}$ and $m_{s}\langle\bar{s} s\rangle / M_{R}^{4}$ for the radial excitations. These scalar radial excitations are also almost degenerated with the pseudoscalar $\pi(1300)$ and $K(1460)$ also indicating the restoration of the spontaneous breaking of the $S U(3)_{L} \times S U(3)_{R}$ chiral symmetry broken by the quark condensate at higher scale ${ }^{14}$. Within such observations, one can expect that these scalar states are $\bar{q} q$ states $^{15}$.

\section{The $I=0$ scalar mesons}

There are proliferations of these states from the data $[1,4]: f_{0}(1370), f_{0}(1500), f_{0}(1710)$ and $f_{0}(1790)$. Using the previous symmetry restorations, one may expect that the 1 st radial excitation of the $(\bar{u} u+\bar{d} d)$ and $\bar{s} s$ unmixed states should be in the range of 1400 $\mathrm{MeV}$, which are in the vicinity of the $f_{0}(1370)$ and $f_{0}(1500)$ being good experimental candidates. The 2 nd radial excitations are expected to be in the range of the $\pi(1800)$ where good experimental candidates are the $f_{0}(1710)$ and $f_{0}(1790)$. Within these observations in mind, we shall interpret the different data given by BES [4]:

- The $f_{0}(1370)$ and $f_{0}(1710)$ are respectively produced through the OZI forbidden $J / \psi \rightarrow \phi \pi^{+} \pi^{-}$and allowed $J / \psi \rightarrow \omega K^{+} K^{-}$, processes [4], while the latter is also produced from the glueball filter process $J / \psi \rightarrow \gamma K^{+} K^{-}$. These features can indicate that they can have an important gluonium component in their wave function. To a first approximation and iteration, we assume that these two states result from the mixing 16 :

$$
\left(\begin{array}{l}
f_{0}(1370) \\
f_{0}(1710)
\end{array}\right)=\left(\begin{array}{cc}
\cos \theta_{S}^{\prime} & \sin \theta_{S}^{\prime} \\
-\sin \theta_{S}^{\prime} & \cos \theta_{S}^{\prime}
\end{array}\right)\left(\begin{array}{c}
\sigma_{B}^{\prime} \\
S_{2}^{\prime}+\phi_{S}^{\prime} S_{3}^{\prime}
\end{array}\right)
$$

which is a replica of the mixing among ground states in Eq. (57). We fix the coupling of the $\bar{q} q$ radial excitations by assuming that they behave like the one of the pion and $\pi(1300)$, namely:

$g_{S_{2,3}^{\prime} \bar{P} P} \approx\left(\frac{M_{S_{2,3}^{\prime}}}{M_{S_{2,3}}}\right)^{n} g_{S_{2,3} \bar{P} P}$,

\footnotetext{
${ }^{14}$ Similar though qualitative arguments have been given in [64]. ${ }^{15}$ Similar conclusions have been also independently reached in [65] using FESR.

${ }^{16}$ Some other alternative mixing schemes above $1 \mathrm{GeV}$ have been also proposed $[12,15],[66,50],[67,68]$.
}

where $P \equiv \pi, K ; n \simeq 2$. Then, we deduce:

$$
\begin{aligned}
g_{S_{2}^{\prime} \pi^{+} \pi^{-}} & \simeq 2 g_{S_{2}^{\prime} K^{+} K^{-}} \simeq 4.8 \mathrm{GeV}, \\
g_{S_{3}^{\prime} K^{+} K^{-}} & \simeq 6.4 \mathrm{GeV} .
\end{aligned}
$$

Using the experimental input from BES [4]:

$$
\begin{aligned}
\Gamma\left(f_{0}(1370 \rightarrow \pi \pi)\right. & \simeq(265 \pm 40) \mathrm{MeV} \\
\frac{\Gamma\left(f_{0}(1370 \rightarrow \bar{K} K)\right.}{\Gamma\left(f_{0}(1370 \rightarrow \pi \pi)\right.} & \simeq(0.08 \pm 0.08)
\end{aligned}
$$

one can fix the two angles to be:

$\theta_{S}^{\prime} \simeq 75^{0} \quad \phi_{S}^{\prime} \simeq-0.55$

The solution indicates that the $f_{0}(1370)$ contains more $(\bar{u} u+\bar{d} d)$ than a gluonium, while the $f_{0}(1710)$ contains more glue and $\bar{s} s$ than $(\bar{u} u+\bar{d} d)$, which is in line with general trends. From this analysis, we predict:

$g_{f_{0}(1710) \pi^{+} \pi^{-}}^{t h} \approx 2.8 \mathrm{GeV}$,

$g_{f_{0}(1710) K^{+} K^{-}}^{t h} \approx 4.3 \mathrm{GeV}$,

which lead to the widths:

$$
\begin{gathered}
\Gamma\left[f_{0}^{t h}(1710) \rightarrow \pi^{+} \pi^{-}\right] \approx 90 \mathrm{MeV}, \\
\Gamma\left[f_{0}^{t h}(1710) \rightarrow K^{+} K^{-}\right] \approx 176 \mathrm{MeV} .
\end{gathered}
$$

The results are of the order of the BES data, which are respectively $\leq 16 \mathrm{MeV}$ and $125 \mathrm{MeV}$ [4], but are not yet quite satisfactory. As the $f_{0}^{t h}(1710)$ contains more glue after the mixing, it is natural that it will mix with the glueball $G(1500)$ with the theoretical parameters obtained previously.

- Therefore, in the 2nd step analysis, we consider that the observed $f_{0}(1710)$ and $f_{0}(1500)$ result from the mixing between $f_{0}^{t h}(1710)$ and $G(1500)$.

- The $f_{0}(1500)$ is produced in the gluonium filter process $J / \psi \rightarrow \gamma \pi^{+} \pi^{-}$, and it is needed for improving the fit of the OZI violating $J / \psi \rightarrow \phi \pi^{+} \pi^{-}$process, but has a small effect in the $J / \psi \rightarrow \phi \bar{K} K$ process [4]. On the other, one expects from previous sections that the $G(1500)$ couplings to $\pi \pi$ and $\bar{K} K$ are small as the vertex sum rule is almost saturated by the $\sigma_{B}$, but we do not have a precise quantitative control of such couplings. However, one can check that the model cannot explain simultaneously the small $f_{0}(1500) \rightarrow K^{+} K^{-}$ and $f_{0}(1710) \rightarrow \pi^{+} \pi^{-}$widths.

- A solution to this problem may be given by the mixing of the previous two states with the $f_{0}(1790)$ and some 2nd radial excitations of the $\bar{q} q$ states expected to be in this range of energy ${ }^{17}$. The $f_{0}(1790)$ may contain more gluon in its wave function as it is produced in the glueball filter channel $J / \psi \rightarrow \gamma \pi^{+} \pi^{-}$ and in the OZI violating $J / \psi \rightarrow \phi \pi^{+} \pi^{-}$process, while it also decays into $4 \pi$. The smallness of the $f_{0}(1790) \rightarrow K^{+} K^{-}$width may come from a destruc-

\footnotetext{
$\overline{17}$ Alternative explanation has been in [69], where it is argued that the glueball $f_{0}(1710)$ has chiral coupling to pairs of Goldstone bosons using perturbative QCD arguments. However, the validity of this result has been questioned in [70] due to instanton contributions.
} 
tive interference between the gluonium and $\bar{q} q$ states ${ }^{18}$.

\section{Conclusions}

Motivated by the various recent experimental progresses for producing scalar mesons, we have proposed new results and updated previous predictions of $[15,42,34]$ :

- The unusual wrong splitting between the $a_{0}(980)$ and $\kappa(900)$ being respectively a $\bar{u} d$ and $\bar{u} s$ mesons can be understood from QSSR using the present value of $\bar{m}_{s}$.

- The maximal meson-gluonium mixing for the $\sigma(600)$ and $f_{0}(980)$ appears to be supported by the present data.

- The $a_{0}(\bar{u} d), \kappa(\bar{u} s), \sigma(600)$ and $f_{0}(980)$ mesons appear to complete the scalar nonet below $1 \mathrm{GeV}$.

- The productions of the $I=0$ mesons above $1 \mathrm{GeV}$ through the OZI violating $J / \psi \rightarrow \phi \pi^{+} \pi^{-}, D_{s} \rightarrow 3 \pi$ decays and $J / \psi \rightarrow \gamma S$ glueball filter processes may indicate that most of these $I=0$ mesons have important gluonium in their wave functions, where a simple meson-gluonium mixing scheme can explain the general features of the data.

- Our results suggest that the $f_{0}(1370)$ is mostly $\bar{q} q$, while the $f_{0}(1500), f_{0}(1710)$ and $f_{0}(1790)$ have significant gluonium component in their wave functions. Tests of these results can be provided by the measurements of the pure gluonium $\eta^{\prime} \eta$ and $4 \pi$ specific $U(1)_{A}$ decay channels.

We conclude that, after about a $1 / 4$ century study, we still remain with more questions than answers on the true nature of scalar mesons.

\section{Acknowledgements}

It is a pleasure to thank Jean-Marc Richard for some comments on the preliminary draft.

\section{REFERENCES}

1. PDG2005, S.E. Eidelman et al., Phys. Lett. B 592 (2004) 1 .

2. For a review prior 2001, see ee e.g.: L. Montanet, Nucl. Phys. Proc. Suppl. 86 (2000) 381; talk given at the Gribov Memory, WSC 2001; U. Gastaldi, Rencontres de Physique de la Vallée d'Aoste, 27 Feb-4 March (2000).

3. WA102 Collaboration, D. Barberis et al. , Phys. Lett. B 479 (2000) 59 [hep-ex/0003033].

4. BES Collaboration, J. Z. Bai et al. , hep ex/0307058 ; M. Ablikim et al. , hep-ex/0406038; hep-ex/0409007; hep-ex/0411001; For a review, see e. g. S. Fang, hep-ex/0509034.

5. E791 Collaboration, I. Bediaga, talk given at QCD05, Montpellier 4 - 8 July 2005 ; Nucl. Phys. Proc. Suppl. 121 (2003) 104.

\footnotetext{
${ }^{18}$ An alternative explanation of the $f_{0}(1790)$ width is based on the restoration of chiral symmetry which favours the previous OZI violation process production of the $f_{0}(1790)$ [64]. However, the author does not explain the absence of its $K^{+} K^{-}$decay.
}

6. FOCUS Collaboration, A. Massafferi, talk given at QCD05, Montpellier 4-8 July 2005.

7. KLOE Collaboration, A. Aloisio et al., Phys. Lett. B 537 (2002) 21; B 536 (2002) 209.

8. SND Collaboration, M.N. Achasov et al., Phys. Lett. B 479 (2000) 53; B 485 (2000) 349.

9. CMD2 Collaboration, R.R. Akhmetshin et al., Phys. Lett. B 462 (1999) 380.

10. BELLE Collaboration, M. Nakao, talk given at QCD05, Montpellier 4-8 July 2005.

11. J. Pelaez and F.J. Yndurain, hep-ph/0510216; R. Kaminski, hep-ph/0510218.

12. V.V. Anisovich, hep-ph/0510409; V.V. Anisovich and A.V. Sarantsev, Eur. Phys. J. A 16 (2003) 229; V.V. Anisovich, D.V. Bugg and A.V. Sarantsev, Phys. Rev. D 58 (1998) 111503.

13. For reviews, see e.g.: S. Narison, QCD as a theory of hadrons, Cambridge Monogr. Part. Phys. Nucl. Phys. Cosmol. 17 (2002) 1 [hep-ph/0205006]; World Sci. Lect. Notes Phys. 26, (1989) 1-529; Riv. Nuov. Cim. 10 N2 (1987) 1; Phys. Rept. 84 (1982) 263.

14. For reviews, see e.g., S. Narison, Nucl. Phys. Proc. Suppl. 121 (2003) 131; Nucl. Phys. Proc. Suppl. 96 (2001) 244; Nucl. Phys. Proc. Suppl. 23 B (1991) 280; Nucl. Phys. A 675 (2000) 54c; hep$\mathrm{ph} / 0009108$.

15. S. Narison, Nucl. Phys. B 509 (1998) 312; Nucl. Phys. Proc. Suppl. 64 (1998) 210.

16. S. Narison, Z. Phys. C 22 (1984) 161; Phys. Lett. B 125 (1983) 501.

17. C. Morningstar and M. J. Peardon, nucl - th / 0309068 ; G. Bali et al. , Phys. Rev. D 62 (2000) 054503; A. Hart and M. Teper, Phys. Rev. D 65 (2002) 34502 ; C. Michael et al. , hep-lat/9909036

18. T. Kunihiro et al., hep-ph/0310312.

19. S. Narison, N. Paver, E. de Rafael and D. Treleani, Nucl. Phys. B 212 (1983) 365.

20. For recent reviews, see e.g.: S. Narison, hepph/0202200, Nucl. Phys. (Proc. Suppl.) B 86 (2000) 242 .

21. K. Chetyrkin and C. Sturm, talk given at QCD05, Montpellier 4-8 July 2005.

22. K. Chetyrkin, S. Narison and V.I. Zakharov, Nucl. Phys. B 550 (1999) 353; S. Narison, Phys. Lett. B 300 (1993) 293.

23. For reviews, see e. g. V. A. Zakharov, hep ph/0509114; F.V. Gubarev, M.I. Polikarpov and V. I. Zakharov, hep-th/9812030; S. Narison, hepph/0508259.

24. M. Jamin, J. A. Oller and A. Pich, Eur. Phys. J. C 24 (2002) 237.

25. S. Narison, hep-ph/0510108.

26. S. Narison, Nucl. Phys. B 182 (1981) 59.

27. T. Schaefer and E. V. Shuryak, Rev. Mod. Phys. 70 (1998) 423.

28. H.G. Dosch and S. Narison, Nucl. Phys. Proc. Suppl. 121 (2003) 114.

29. G. Launer, S. Narison and R. Tarrach, Z. Phys. C 26 (1984) 433; R.A. Bertlmann et al., Z. Phys. 
C 39 (1988) 231; Y. Chung et al., Z. Phys. C 25 (1984) 151; H.G. Dosch, M. Jamin and S. Narison, Phys. Lett. B 220 (1989) 251.

30. S. Narison, Phys. Lett. B 216 (1989) 191.

31. K. Maltman, hep-ph/9906267.

32. S. Narison and N. Paver, Phys. Lett. B 158 (1985) 153.

33. S. Narison, Phys. Lett. B 175 (1986) 88.

34. A. Bramon and S. Narison, Mod. Phys. Lett. A 4 (1989) 1113.

35. R.L. Jaffe, Phys. Rev. D 15 (1977) 267; D 17 (1978) 1444.

36. J.I. Latorre and P. Pascual, Jour. Phys. G11 (1985) L231.

37. M. Alford and R.L. Jaffe Nucl. Phys. B 509 (1998) 312.

38. T.V. Brito, F.S. Navarra, M.Nielsen, M.E. Bracco, Phys. Lett. B 608 (2005) 69.

39. P. Di Vecchia and G. Veneziano, Nucl. Phys. B 171 (1981) 253.

40. See e.g.: Kyoto Workshop on the $\sigma$ meson, KEK Proceedings 2000-4.

41. D. Black, M. Harada and J. Schechter, Nucl. Phys. Proc. Suppl. 121 (2003) 95; J. Ellis and J. Lanik, Phys. Lett. B 150 (1985) 289; H. Gomm et al., Phys. Rev. D 33 (1986) 801; D. Ebert et al., hepph/0007131; A. Boduylov and V. Novozhikov, Desy report 90-151 (1990); G. Mennessier, Z. Phys. C 16 (1983) 241.

42. S. Narison and G. Veneziano, Int. J. Mod. Phys. A 4, 11 (1989) 2751.

43. V.A. Novikov et al., Nucl. Phys. B 191 (1981) 301.

44. S. Narison, Phys. Lett. B 361 (1995) 121; Phys. Lett. B 387 (1996) 162.

45. S. Narison, G. Shore and G. Veneziano, Nucl. Phys. B 433 (1995) 209; Nucl. Phys. B 546 (1999) 235; For a recent review, see e.g. S. Narison, hep$\mathrm{ph} / 0601066$.

46. T.G. Steele, D. Harnett and G. Orlandini, hepph/0308074.

47. H. Forkel, hep-ph/0312049.

48. T.G. Steele, D. Harnett and G. Orlandini, hepph/0108232.

49. V.A. Novikov et al. Nucl. Phys. B 165 (1980) 67.

50. P. Minkowski and W. Ochs, Eur. Phys. J. C 9 (1999) 283; Nucl. Phys. Proc. Suppl. 121 (2003) 119; Nucl. Phys. Proc. Suppl. 121 (2003) 121.

51. E. Witten, Nucl. Phys. B156 (1979) 269; G. Veneziano, Nucl. Phys. B159 (1979) 213.

52. A. Di Giacomo, Nucl. Phys. Proc. Suppl. B 23 (1991) 191.

53. F. Gilman and R. Kauffman, Phys. Rev. D 36 (1987) 2761; L. Montanet, Non-Perturbative Methods Conf., Montpellier, (WSC 1985).

54. G. Mennessier, S. Narison and N. Paver, Phys. Lett. B 158 (1985) 153; N. Pak, S. Narison and N. Paver, Phys. Lett. B 147 (1984) 162.

55. M.R. Pennington, hep-ph/0604212.

56. N.N. Achasov and A.V. Kiselev, hep-ph/0512047.

57. A. Bramon et al., hep-ph/000818; Yu. Kalashnikova et al., nucl-th/0512028; T. Barnes, Phys.
Lett. B 165 (1985) 434; N. Achasov and G.N. Shestakov, Phys. Rev. D 72 (2005) 013006.

58. A. Deandrea et al., hep-ph/0012120.

59. S. S. Gershtein , A. A. Likhoded and Y. D. Prokoshkin, Z. Phys. C24 (1984) 305.

60. H. G. Dosch et al., hep-ph/0203225; M. Nielsen et al., Nucl. Phys. Proc. Suppl. 121 (2003) 110.

61. L.S. Kisslinger, hep-ph/0103326.

62. M. Nielsen, F.S. Navarra and I. Bediaga, Nucl. Phys. Proc. Suppl. 133 (2004) 178.

63. P. Minkowski and W. Ochs, hep-ph/0511126.

64. L.Ya. Glozman, hep-ph/0507206.

65. A.L. Kataev, Phys. Atom. Nucl. 68 (2005) 567; hep-ph/0406305 and references threin.

66. F. Giacosa, Th. Gutsche, V.E. Lyubovitskij and A. Faessler, hep-ph/0504033.

67. C. Amsler and F.E. Close, Phys. Rev. D 53 (1996) 295; D. Weingarten, Nucl. Phys. Proc. Suppl. 73 (1999) 283; C. Amsler, hep-ph/0206104; F.E. Close and A. Kirk, hep-ph/0103173.

68. J. Vijande et al., hep-ph/0508142.

69. M.S. Chanowitz, hep-ph/0506125.

70. Z.F.Zhang, H.Y.Jin, hep-ph/0511252. 